\title{
Acoustic Pulse Thrombolysis Complemented by ECMO Improved Survival in Patients with High-Risk Pulmonary Embolism
}

\author{
Mert Dumantepe ${ }^{1}$ and Cuneyd Ozturk ${ }^{2}$ \\ ${ }^{1}$ Uskudar University \\ ${ }^{2}$ Florence Nightingale Hospital
}

September 29, 2021

\begin{abstract}
Background: The optimal treatment of high-risk PE with cardiac arrest is still controversial although various treatment approaches have been developed and improved. Here, we present a serie of patients with high-risk PE showing hemodynamic collapse, who were successfully treated with extracorporeal membrane oxygenation (ECMO) as an adjunct to EKOS acoustic pulse thrombolysis. Method: From April 2016 to June 2020, 29 patients with high-risk PE with cardiac arrest were retrospectively included. The mean age was $55.3 \pm 9.2$ years. Twelve (41.3\%) patients were female. All patients had cardiac arrest, either as an initial presentation or in-hospital after the presentation. All patients exhibited acute symptoms, computed tomography (CT) evidence of large thrombus burden, and severe right ventricular dysfunction. Primary outcome was all-cause 30-day mortality. Results: Twenty-two patients survived to hospital discharge, with a mean ICU stay of $9.9 \pm 1.6$ days (range, 7 to 22 days) and mean length of hospital stay of $23.7 \pm 8.5$ days (range, 11 to 44 days). Six patients died from refractory shock. Ninety-day mortality was $24.1 \%$ (7/29). The Mean ECMO duration was $3.5 \pm 1.1$ days and the mean RV/LV ratio decreased from 1.31 \pm 0.17 to $0.92 \pm 0.11$ in patients who survived to discharge. The mean tissue plasminogen activator (tPA) dose for survivor patients was $20.5 \pm 1.6 \mathrm{mg}$. Conclusion: Patients with high-risk pulmonary embolism who suffer a cardiac arrest have high morbidity and mortality. APT complemented by ECMO could be a successful treatment option for patients who have high-risk PE with circulatory collapse.
\end{abstract}

\section{Introduction}

Acute pulmonary embolism (PE) can be stratified as high-risk or massive PE, intermediate-risk or submassive PE, and low-risk or non-massive PE, with nearly 300,000 deaths per year attributable to $\mathrm{PE}^{1}$. Right ventricular dysfunction (RVD) and the presence of hemodynamic instability are powerful predictors of poor prognosis of patients with acute $\mathrm{PE}^{2}$. In particular, patients with acute high-risk PE defined as cardiogenic shock and systemic hypotension, are at particularly increased risk of early death, and require emergency treatment to restore circulation. Overall, in-hospital mortality rates for massive PE range from $25 \%$ for patients with cardiogenic shock (CS), to $65 \%$ for those that required cardiopulmonary resuscitation (CPR), while in-hospital mortality rate in stable patients with $\mathrm{PE}$ was $8.1 \%{ }^{3}$.

Although systemic anticoagulation remains the cornerstone of therapy for acute symptomatic pulmonary embolism, the morbidity and mortality associated with high-risk PE and intermediate-high risk PE warrant treatment beyond anticoagulation alone. More aggressive therapeutic options include systemic intravenous thrombolysis (Class IB), surgical embolectomy (Class IC) and catheter-directed thrombolysis needed (CDT) (Class IIa) according to European Society of Cardiology guidelines ${ }^{4}$.

However, the clinical course of high-risk PE can rapidly progress before surgical or catheter-directed treatment. Many patients are not amenable to reperfusion therapies or fail to improve after these treatments due 
to major hemodynamic instability and cardiogenic shock. Based on the ICOPER Registry, two-thirds of patients with massive PE did not receive any thrombolysis or surgical embolectomy ${ }^{5}$. For those patient group, veno- arterial extracorporeal membrane oxygenation (VA-ECMO) is one of the most reliable and quickest way to decrease RV overload, improve RV function and hemodynamic status, restore tissue oxygenation and may be considered as either a bridge to reperfusion therapy such as surgical embolectomy or $\mathrm{CDT}^{6}$.

The general aim of catheter-directed therapy in the setting of hemodynamically compromised patients with acute $\mathrm{PE}$ is to debulk and/or redistribute the obstructive clot rendering it less hemodynamically significant. In recent years, interest has risen in a variety of endovascular strategies based on catheter-based technologies for thrombus removal in patients with high-risk PE. The EKOS? EkoSonic Endovascular System (EKOS, Boston Scientific, USA) is a catheter-based system that uses high frequency, low-energy ultrasound waves to aid in the delivery and uptake of thrombolytic agent within the clot.

Existing data regarding the utility of ECMO in the setting of high-risk PE mainly come from case reports or small series ${ }^{(6-8)}$. The impacts of VA-ECMO in conjunction with EKOS acoustic pulse thrombolysis (APT) on survival have not been investigated in massive PE. Therefore, we present a serie of patients with high risk PE showing hemodynamic collapse that required cardiopulmonary resuscitation, who were successfully treated with VA-ECMO as an adjunct to APT.

\section{Methods}

\section{Patients}

A total of 154 consecutive patients with MPE, initially diagnosed by either computed tomography or echocardiography from April 2016 to June 2020 transferred from emergency department to our catheterization laboratory, were retrospectively evaluated, of whom 29 patients met the criteria for high-risk PE complicated by profound cardiogenic shock or that required CPR $(n=29)$, were enrolled in the study to analyze clinical outcomes. Written informed consent was obtained from each enrolled patient or from the family after the purpose and the risk of the treatment were fully explained. The Institutional Committee of Ethics in Research approved this clinical research project.

\section{Data collection}

A manual review of patient charts was then undertaken to confirm the diagnosis and to obtain preoperative, perioperative, and postoperative variables and outcomes. At hospital or ICU admission, we collected the following information: demographics, presence of venous-thromboembolic risk factors; Simplified Acute Physiology Score (SAPS) $\mathrm{II}^{9}$ and Sequential Organ Failure Assessment (SOFA) score ${ }^{10}$.

During the pre-ECMO period, the inotrope score ${ }^{11}$, defined as dobutamine dose $(\gamma / \mathrm{kg} / \mathrm{min})+$ [norepinephrine dose $(\gamma / \mathrm{kg} / \mathrm{min})+$ epinephrine dose $(\gamma / \mathrm{kg} / \mathrm{min})] \times 100$; cardiopulmonary arrest with its related "low-flow" and "no-flow" situations; and troponin level and blood gas analyses were noted. The echocardiography variables which includes; left ventricular ejection fraction (LVEF), RV/LV dimension ratio and visualization of a pulmonary arterial thrombus were also recorded before ECMO insertion. RV dysfunction (none, mild, moderate, or severe) was recorded according to a preoperative and postoperative transthoracic echocardiogram by an independent cardiologist, who both quantitatively and qualitatively assessed RV function.

The pulmonary angiography was independently reviewed for quantification of pulmonary emboli by one observer who was blinded to the clinical course. We applied an embolic burden scoring system in our study: observer scored main, lobar, right interlobar and segmental pulmonary arteries for the presence of emboli and also graded whether emboli were occlusive ${ }^{12}$.

\section{Indications for VA-ECMO and Management of ECMO Support}

VA-ECMO indications were: acute refractory cardiovascular failure, defined as evidence of tissue hypoxia concomitant with adequate intravascular volume status; severely diminished RV function or LVEF; low car- 
diac index ([?]2.1 L/min/m2); sustained hypotension despite high-dose catecholamine infusion (epinephrine [?]1 $\mathrm{\gamma} / \mathrm{kg} / \mathrm{min}$ or dobutamine [?]20 $\mathrm{\gamma} / \mathrm{kg} / \mathrm{min}+$ norepinephrine [?]1 $\mathrm{\gamma} / \mathrm{kg} / \mathrm{min}$ ).

All patients, regardless of age or comorbid conditions, were considered candidates for this intervention, unless their predicted survival independent of the PE, was less than 1 year. ECMO consist of a centrifugal pump, a hollow fiber oxygenator (Livanova, Sorin Group, Italy) and cannulas (Xenios AG-Medos, Heilbronn Germany). Cannulation was performed under ultrasound guidance, with a $23 \mathrm{~F}$ to $25 \mathrm{~F}$ venous drainage cannula placed in a femoral vein and a $17 \mathrm{~F}$ to $19 \mathrm{~F}$ arterial return cannula placed in a common femoral artery in the contralateral groin whether in the ICU or in the operation room. A $6 \mathrm{~F}$ distal perfusion cannula was placed in the superficial femoral artery as a first step previously, unless the patient was actively requiring cardiopulmonary resuscitation (CPR), to allow for better ultrasound visualization of the superficial femoral artery.

ECMO Flows were maintained in the range of 2 to $4 \mathrm{~L} /$ minute, requiring pump speeds of approximately 3,000 to $4,000 \mathrm{rpm}$, and were adjusted with the goals of reversing acidosis while maintaining arterial saturation and cardiac output. Support flow was also titrated up until the right ventricle was decompressed by TTE and maintain a systemic blood pressure of $90 \mathrm{mmHg}$. However, some pulmonary blood pulmonary flow was maintained to allow for APT. Systemic anticoagulation was maintained with activated clotting time of 180200 seconds by unfractionated heparine infusion. Figure 1. shows one survivor patient who have high-risk $\mathrm{PE}$ and cardiac arrest, stabilized hemodynamically with VA-ECMO; just before catheter-based intervention with EKOS? APT.

\section{Treatment Procedure}

Patients were taken to the hybrid operating room for EKOS? APT after stabilization of hemodynamic status with the support of VA-ECMO. For the placement of the EkoSonic Endovascular System common femoral vein access was established by placement of a bilateral 6 French vascular sheath. Digital subtraction angiography was performed in two bilateral anterior oblique projections with the pigtail diagnostic catheter positioned in the main pulmonary artery. Once the location of a pulmonary arterial thrombus was identified angiographically, the angiographic catheters were exchanged for an EkoSonic infusion device, which was navigated over a 0.035 -inch angled guidewire, such that the distal tip of the EKOS? infusion catheter was positioned at the distal edge of the thrombus under fluoroscopy (Figure 2).

Patients were transferred to the ICU for thrombolytic infusion with tPA administered at a rate of 1 to 1.5 $\mathrm{mg} / \mathrm{hr}$ ( 0.5 to $0.75 \mathrm{mg} / \mathrm{hr}$ through each catheter, in case of bilateral PE). $1 \mathrm{mg}$ bolus tPA per catheter was administered to the patients before the overnight infusion. Normal saline coolant was infused at a rate of 40 $\mathrm{cc} / \mathrm{hr}$, through the coolant port of the EKOS? infusion catheter.

\section{Clinical Outcomes}

The main outcome variables included ECMO weaning, survival to hospital discharge, 90-day survival, and long-term survival. Secondary outcomes included acute kidney injury that required renal replacement therapy, sepsis, ischemic stroke, RV dysfunction at discharge, ECMO-related complications and vascular complications. Furthermore, we calculated patient's SOFA scores at cannulation and day 1, 3, and 7 and the inotrope score; during the ECMO and mechanical ventilation.

Bleeding complications were reported using the GUSTO classification ${ }^{13}$. The number of packs of blood products transfused were also collected.

Statistical analyses

Statistical analysis was performed with Graphpad Instat software (version 3 for Mac; version 11.5, GraphPad Software, Inc., La Jolla, CA, USA). Results are expressed as numbers (\%) or mean \pm standard deviation (range). Continuous variables were compared with Student's t test or the Mann-Whitney U test, as appropriate, whereas categorical variables were compared with chi-square tests. The change from baseline to follow-up of both the RV/LV ratio and the embolic burden score was assessed using one-way, repeated 
measures analysis or variance for respiratory parameters using ANOVA. A p-value $<0.05$ was considered statistically significant.

\section{Results}

\section{Study Population}

During the study period, a total of 154 patients with high-risk PE were identified, of whom 29 patients with high risk PE, complicated by cardiogenic shock or that required CPR were treated with ECMO in conjunction with EKOS? APT. The baseline clinical characteristics of the patients are summarized in Table 1. Twelve of 29 patients placed on ECMO with cardiac arrest were female and mean age $55.3+-9.2$ years. The majority of patients had major transient risk factors for VTE, such as surgery, immobilization, or prolonged bed rest. Nine patients (31\%) had a prior history of VTE, $5(17.2 \%)$ patients have malignancy, 7 (24.1\%) patients have positive hypercoagulable work-up after presentation. The most frequent comorbidities were hypertension $(48.2 \%)$, smoking $(55.1 \%)$, and cardiovascular disease $(20.6 \%)$.

Mean SAPS II and SOFA score were high, respectively, $78.3+-14.5$ and $13.1+-3.8$. All patients required hemodynamic support with vasoactive drugs, resulting in a mean inotrope score of $117+-16.3$ (20-197) $\mu \mathrm{g} / \mathrm{kg} / \mathrm{min}$ at ECMO cannulation. The mean troponin level was $2.12 \pm 0.37 \mathrm{ng} / \mathrm{mL}$ and the mean pro-B-type natriuretic peptide (pro-BNP) level was $3255 \pm 1170 \mathrm{pg} / \mathrm{L}$. Pre-ECMO mean blood pressure, $\mathrm{pH}$ and blood lactate was, respectively: $55.6 \pm 7.7 \mathrm{mmHg}, 7.11 \pm 0.13$ and $12.5 \pm 2.6 \mathrm{mmol} / \mathrm{L}$ (Table 2). Transthoracic or transesophageal echocardiography visualized major RV dilation in all patients (mean RV/LV dimensions ratio $1.7 \pm 0.3[1.1-1.9]$ ). On computed tomography (CT) scan, 11 patients (38\%) had saddle PE and an additional six patients (20.6\%) had clot-in-transit in the right atrium. Pulmonary angiographic findings showed that a filling defect or total occlusion was observed in all 29 the patients at the proximal lobar or lobular arteries and additionally 11 of them had massive thrombus in the main pulmonary arteries. Figure $3 \mathrm{~A}$ showed large saddle thrombus in main pulmonary artery in CT with contrast enhancement.

\section{ECMO Cannulation and Management}

All patients had cardiac arrest, either as an initial presentation or in-hospital after presentation. The patients required CPR before cannulation for ECMO, with a range of 3 to 50 minutes of CPR. 8 of 29 patients (27.5\%) were placed on ECMO during CPR, and the remaining patients were placed on ECMO after successful CPR at an outside or inside of the hospital. Six patients had in-hospital onset, with diagnosis during the hospitalization of acute myocardial infarction, post abdominal surgery and severe pneumonia. All patients had VA- ECMO, 26 patients had femoral, three patients had central, respectively. Mean ECMO duration was $3.5 \pm 1.1$ days for survivor patients, ranging from 2-11 days.

All patients underwent APT therapy using the EKOS? system. No other catheter-based procedures (e.g. Percutaneous mechanical thrombectomy) were performed. The mean tissue plasminogen activator (tPA) dose for survivor patients was $20.5+-1.6 \mathrm{mg}$ and the mean infusion time was $16.2+-3.1$ hours (Table 3).

End-point Analysis, complications and 90-Day Survival

The preoperative mean embolic burden score was $19.5+-4.7$ and decrease to $1.4+-2.1$ for survivor patients (Table 3). Follow-up contrast-enhanced CT studies were performed after $76+-12$ hours. The patients saddle pulmonary embolisms were clearly disappeared after successful treatment in the CT follow-up ( Figure 3B). For survivor patients, the mean RV/LV ratio decreased from $1.31+-0.17$ to $0.92+-0.11$ at follow-up $(\mathrm{p}<0.001)$ and the mean right ventricular end diastolic diameter was reduced from $54.3+-6.6$ to 41.5 +$5.3 \mathrm{~mm}$.

Table 3 reports ECMO-related complications and short-term outcomes according to 90-day status. Seven of 29 patients (20.6\%) experienced at least one minor ECMO-related complication; 7 (24.1\%) patients had a moderate hemorrhage classified as GUSTO [?]2 with a mean of 2.2 +- 0.3 (1-4) packed red-cell and 3.1 +- $0.8(1-5)$ fresh-frozen plasma units transfused. Four of 29 patients (13.7\%) experienced acute kidney 
injury that required renal replacement therapy during the hospitalization. However, only 2 of those patients required dialysis after discharge, which was discontinued during follow-up.

Other complications included: ischemic stroke in three patients, with two recovering to normal neurological function at hospital discharge and 1 patient develop hypoxic-ischemic encephalopathy, and two surgical cannulas related, wound-infection debridement. In our patient series, the patients did not demonstrate any signs of acute limb ischemia, the distal perfusion cannula prevented acute limb ischemia.

Twenty-two patients survived to hospital discharge, with a mean ICU stay of $8.9+-1.6$ days (range 7 to 22 ) and mean length of hospital stay of $23.7+-8.5$ days (range, 11 to 44 days). Six patients died from refractory shock despite ECMO support and cardiac arrest-related multiorgan failure. One patient died after successful ECMO weaning with PE recurrence 55 days post- ECMO removal despite adequate anticoagulation. Ninetyday mortality was $24.1 \%(7 / 29)$ and one-year mortality was $27.5 \%(8 / 29)$. Compared with patients who died within 90 days, it is worth noting that 90-day survivors had

significantly lower inotrope scores 24 hours post ECMO cannulation and lower SAPS II and SOFA scores on ECMO day 1 (Table 3 ).

\section{Discussion}

Pulmonary embolism is the most serious clinical presentation of venous thromboembolism and is associated with significant morbidity and mortality. In particular, patients with acute high-risk PE defined as cardiogenic shock and systemic hypotension, are at particularly increased risk of early death, and require emergency treatment to restore circulation ${ }^{14}$.

Current guidelines for high-risk PE treatment recommend primary reperfusion therapy and the option of ECMO. However, these recommendations might not be optimal for patients with poor prognoses who are in cardiogenic shock or require CPR. Patients with collapsed high-risk PE have an extremely high-risk mortality rate because conventional CPR including chest compression is usually not effective due to right ventricle outflow obstruction. The in-hospital mortality of high-risk PE requiring CPR may reach $65 \% 15$.

The majority of deaths in patient presenting with circulatory collapse occur within the first hour after the presentation, and rapid therapeutic action is therefore essential to save lives ${ }^{(4,5)}$. ECMO is the most convenient circulatory assist method widely available and is the alternative of choice for treating patients with high-risk PE with circulatory collapse ${ }^{16}$. Resuscitation guidelines suggest therapy with thrombolytics in patients with pulmonary embolism and cardiac arrest; but, in case of refractory cardiac arrest, because of the inherent risk of bleeding of systemic thrombolysis, no further survival options can be offered to patients with treatment failure till now ${ }^{17}$.

Catheter based interventions is recommended for patients with cardiogenic shock due to high-risk PE, and its equivalent to surgical embolectomy ${ }^{18}$. Furthermore, catheter interventions during CPR has been performed successfully in a small number of patients ${ }^{19}$.

ECMO is recommended to provide pulmonary and circulatory support for the emergency treatment of patients with massive pulmonary embolism and cardiac arrest. It provides the ability to decompress the acutely overloaded right atrium and ventricle, increase the aortic pressure and myocardial blood flow, reverse ischemia and consequently improve the right ventricular function. Many publications have demonstrated that ECMO has a role as a bridge to percutaneous coronary intervention in patients with acute coronary syndrome and cardiogenic shock, as well as in the treatment of cardiogenic shock itself ${ }^{20-21)}$. Similarly, in patients with high-risk PE, ECMO can function as an adjunct to anticoagulation as a bridge to invasive management, such as surgical embolectomy or CDT; or, as post-procedural support for patients undergoing these therapies. Yusuff and colleagues, conducted a systematic review of the literature on ECMO in patients with high-risk PE. There were no randomized control trials, however, they reviewed over 20 years of case reports on the topic and found an overall survival of $70.1 \%$. They did note that those who had ECMO initiated while in cardiac arrest had an overall higher mortality compared with those who never experienced such an event ${ }^{22}$. 
To our knowledge, our series represents the largest review of VA-ECMO as an adjunct to EKOS? APT for high-risk PE treatment reported in the literature. Although multiple case reports and series have previously demonstrated the use of ECMO for massive PE, published mortality rates have been prohibitive over long study periods ${ }^{(23,24)}$. Hashiba et al have reported that the ECMO survival rate at discharge was $83.3 \%$. The outcome could be attributable to lower proportions of patients with cardiac arrest, compared with our study population ${ }^{25}$. Maggio et al have found that the survival rate of ECMO for massive PE was 13 of 21 or $62 \%$. However, only 8 of 21 patients experienced cardiac arrest, which was a smaller percentage than in our study ${ }^{26}$.

Recently, Meneveau et al published the largest multicenter series of high-risk PE patients

with refractory cardiogenic shock or cardiac arrest undergoing $\mathrm{ECMO}^{27}$. The overall mortality rate was $61.5 \%$ in high-risk PE patients who receive ECMO, especially in those with failed fibrinolysis and in those with no reperfusion treatment. In patients undergoing ECMO, 30-day mortality was $76.5 \%$ (13/17) for ECMO + fibrinolysis and 77.8\% (14/18) for ECMO alone. They concluded that ECMO does not appear justified as a stand-alone treatment strategy in high-risk PE patients with cardiogenic shock, but shows promise as a complement to another reperfusion treatment such as surgical embolectomy or CDT. Conversely to our study, Meneveau and colleagues did not assess the potential role of catheter directed treatments in association with ECMO in their patient cohort. There may be unstable, severely ill patients who are not suitable for the intervention, or center who do not have experienced interventionalist or surgical facilities on site. However, they also concluded that, catheter-based interventions could be a useful alternative that would make it possible to reduce the time delay between onset and pulmonary reperfusion, by combining the initiation of circulatory assistance with percutaneous treatments within the same procedure. Recent data have shown promising results regarding the safety and efficacy of this type of procedure in patients with intermediate and/or high-risk $\mathrm{PE}^{28}$.

Dolmatova and colleagues described a series of 5 patients over 5 years, with a $60 \%$ survival. In that group's series, ECMO was used as a salvage therapy in 4 of 5 patients after other therapies failed, with a $50 \%$ mortality rate in this subset ${ }^{29}$. They also concluded that ECMO may be reasonable to initiate in patients with a highrisk PE who would otherwise be expected to die. However, our study contradicts the opinion of using ECMO as a salvage therapy. Because, with early and aggressive use of ECMO in conjunction with EKOS? APT, we demonstrated a $75.9 \%$ survival rate for patients with a high-risk PE who had cardiopulmonary arrest and required CPR. We believe that early restoration of adequate perfusion limits ischemia, mitigates permanent end-organ damage, and prevents initial or recurrent cardiac arrest in this critically ill group of patients.

The clinical status of the survivor patients in our study, markedly improved from time of cannulation to definitive intervention. Lactate, $\mathrm{pH}$, and bicarbonate levels all normalized. We also found that, in nearly all cases, patients who survived to hospital discharge had normal RV parameters on follow-up echocardiogram. Patients required a considerably lower dose of inotropic/vasopressor agents, and SOFA scores notably improved, indicating a lower predicted intensive care unit rate of mortality after successful catheter-based intervention with the support of VA- ECMO.

APT with EKOS? system enhances CDT by accelerating the fibrinolytic process via the application of ultrasound. Improving the efficiency of the thrombolytic process reduces the treatment time and total lytic dose delivered. The risk of an associated bleeding complication, which is extremely increased by the concomitant ECMO procedure, is, therefore, reduced ${ }^{31}$. Kuo et al reported a high pooled clinical success rate of $86.5 \%$ in 594 patients treated with various CDT techniques ${ }^{30}$. The risks of major and minor complications were low at $2.4 \%$ and $7.9 \%$, respectively.

Although ECMO may be helpful in many patients who have a low chance of surviving high-risk PE without hemodynamic support, it does come with complications, in particular, major bleeding. Despite the highrisk of bleeding due to ECMO and using thrombolytics, our major bleeding rate was lower than reported elsewhere ${ }^{(6,32)}$. In our study only $8(27.5 \%)$ patients had a moderate hemorrhage classified as GUSTO [?]2 with a median of 2 packed red-cell and 3 fresh-frozen plasma units transfused. 
An important consideration in percutaneous femoral access for V-A ECMO is lower-extremity acute limb ischemia. In a retrospective study of 43 patients with femoral arterial cannulae on ECMO, there were no cases of limb ischemia in patients with prophylactically placed SFA distal perfusion cannulae; $21 \%$ of patients without such cannulae did develop limb ischemia ${ }^{33}$. Our practice is to routinely place a prophylactic distal perfusion cannula in the SFA. Therefore, in our patient series, the patients did not demonstrate any signs of acute limb ischemia.

The results of our study require carefully interpretation. First the study was retrospective and single center experience. Second, the present study did not have a comparative group of patients who were not undergoing APT with the support of V-A ECMO. Furthermore, catheter-based interventions necessitate skilled physicians as a member of interdisciplinary team required for successful treatment of high-risk PE.

\section{Conclusion}

High-risk PE imposes a high level of morbidity and mortality. Treatment of this entity, requires diverse, aggressive, expeditious, and multidisciplinary approaches that are tailored to

meet patient-specific risks and needs. ECMO is a useful clinical tool, which can be implemented to provide pulmonary and circulatory support for the emergency treatment of patients with circulatory collapse. EKOS? Acoustic pulse thrombolysis concomitantly with V-A ECMO is providing definitive and live saving treatment in patient with high-risk $\mathrm{PE}$ and cardiogenic shock.

\section{References}

1. Tapson VF: Acute pulmonary embolism. N Engl J Med 2008; 358:1037-1052.

2. Wood KE: Major pulmonary embolism: Review of a pathophysiologic approach to the golden hour of hemodynamically significant pulmonary embolism. Chest 2002;121: 877-905.

3. Agnelli G, Becattini C. Acute pulmonary embolism. N Engl J Med. 2010; 363(3):266-

74.

4. Konstantinides SV, Meyer G, Becattini C, et al. 2019 ESC Guidelines for the diagnosis and management of acute pulmonary embolism developed in collaboration with the European Respiratory Society (ERS). Eur Heart J. 2020 Jan 21;41(4):543-603.

5. Kucher N, Rossi E, De Rosa M, Goldhaber SZ. Massive pulmonary embolism. Circulation. 2006;113(4):577-82.

6. Corsi F, Lebreton G, Brechot N, et al. Life-threatening massive pulmonary embolism rescued by venoarterial-extracorporeal membrane oxygenation. Crit Care. 2017;21(1):76.

7. Omar HR, Miller J, Mangar D, Camporesi EM. Experience with extracorporeal

membrane oxygenation in massive and submassive pulmonary embolism in a tertiary

care center. Am J Emerg Med 2013; 31:1616-1617

8. Malekan R, Saunders PC, Yu CJ, et al. Peripheral extracorporeal membrane oxygenation: comprehensive therapy for high-risk massive pulmonary embolism. Ann Thorac Surg 2012; 94:104-108.

9. Le Gall JR, Lemeshow S, Saulnier F. A new Simplified Acute Physiology Score (SAPS II) based on a European/North American multicenter study. JAMA. 1993;270(24):2957-63.

10. Vincent JL, Moreno R, Takala J,et al. The SOFA (Sepsis-related Organ Failure Assessment) score to describe organ dysfunction/failure. On behalf of the Working Group on Sepsis-Related Problems of the European Society of Intensive Care Medicine. Intensive Care Med. 1996;22(7):707-10. 
11. Wernovsky G, Wypij D, Jonas RA, et al. Postoperative course and hemodynamic profile after the arterial switch operation in neonates and infants. A comparison of low-flow cardiopulmonary bypass and circulatory arrest. Circulation. 1995; 92(8): 2226-35

12. Araoz PA, Gotway MB, Harrington JR, Harmsen WS, Mandrekar JN. Pulmonary embolism: prognostic CT findings. Radiology. 2007 Mar; 242(3): 889-97.

13. The GUSTO Angiographic Investigators. The effects of tissue plasminogen activator, streptokinase, or both on coronary-artery patency, ventricular function, and survival after acute myocardial infarction. N Engl J Med. 1993; 329 (22):1615-1622

14. Giri J, Sista AK, Weinberg I, et al. Interventional Therapies for Acute Pulmonary Embolism: Current Status and Principles for the Development of Novel Evidence: A Scientific Statement from the American Heart Association. Circulation. 2019 Nov 12;140(20):774-801

15. Kabrhel C, Rosovsky R, Channick R. A multidisciplinary pulmonary embolism response team: initial 30-month experience with a novel approach to delivery of care to patients with submassive and massive pulmonary embolism. Chest 2016; 150: 384-393

16. Maggio P, Hemmila M, Haft J, Bartlett R. J. Extracorporeal life support for massive pulmonary embolism. Trauma. 2007 Mar;62(3):570-6.

17. Nolan JP, Soar J, Zideman DA, et al. European Resuscitation Council Guidelines for Resuscitation 2010 Section 1. Executive summary. Resuscitation. 2010; 81: 1219-1276.

18. Jaff MR, McMurtry MS, Archer SL, et al. Management of massive and submassive pulmonary embolism, iliofemoral deep vein thrombosis, and chronic thromboembolic pulmonary hypertension: a scientific statement from the American Heart Association. Circulation. 2011 Apr 26;123(16):1788-830.

19. Fava M, Loyola S, Bertoni H, Dougnac A. Massive pulmonary embolism: percutaneous mechanical thrombectomy during cardiopulmonary resuscitation. J Vasc Interv Radiol. 2005 Jan;16(1):119-23.

20. Sheu JJ, Tsai TH, Lee FY, et al. Early extracorporeal membrane oxygenator-assisted primary percutaneous coronary intervention improved 30-day clinical outcomes in patients with ST-segment elevation myocardial infarction complicated with profound cardiogenic shock. Crit Care Med 2010; 38: 1810-1817.

21. Sakamoto S, Taniguchi N, Nakajima S, et al. Extracorporeal life support for cardiogenic shock or cardiac arrest due to acute coronary syndrome. Ann Thorac Surg 2012; 94: 1-7.

22. Yusuff HO, Zochios V, Vuylsteke A. Extracorporeal membrane oxygenation in acute massive pulmonary embolism: a systematic review. Perfusion 2015;30(8): 611-616

23. Hsieh PC, Wang SS, Ko WJ, Han YY, Chu SH. Successful resuscitation of acute massive pulmonary embolism with extracorporeal membrane oxygenation and open embolectomy. Ann Thorac Surg 2001; 72:266-7.

24.Davies MJ, Arsiwala SS, Moore HM, Kerr S, Sosnowski AW, Firmin RK. Extracorporeal membrane oxygenation for the treatment of massive pulmonary embolism. Ann Thorac Surg 1995; 60:1801-3.

25. Hashiba K, Okuda J, Maejima N, et al. Percutaneous cardiopulmonary support in pulmonary embolism with cardiac arrest. Resuscitation. 2012;83(2):183-7.

26. Maggio P, Hemmila M, Haft J, Bartlett R. Extracorporeal life support for massive pulmonary embolism. J Trauma. 2007;62(3):570-6.

27. Meneveau N, Guillon B, Planquette B, et al Outcomes after extracorporeal membrane oxygenation for the treatment of high-risk pulmonary embolism: a multicentre series of 52 cases. Eur Heart J. 2018 Dec 14;39(47):4196-4204.

28. Kucher N, Boekstegers P, Muller OJ, et al. Randomized, controlled trial of ultrasound-assisted catheterdirected thrombolysis for acute intermediate-risk pulmonary embolism. Circulation 2014; 129:479-486. 
29. Dolmatova EV, Moazzami K, Cocke TP, et al. Extracorporeal membrane oxygenation in massive pulmonary embolism. Heart Lung 2017; 46:106-9.

30. Kuo WT, Gould MK, Louie JD, Rosenberg JK, Sze DY, Hofmann LV. Catheter-directed therapy for the treatment of massive pulmonary embolism: systematic review and meta-analysis of modern techniques. J Vasc Interv Radiol. 2009 Nov;20(11):1431-40

31. Dumantepe M, Uyar I, Teymen B, Ugur O, Enc Y. Improvements in pulmonary artery pressure and right ventricular function after ultrasound-accelerated catheter-directed thrombolysis for the treatment of pulmonary embolism. J Card Surg. 2014 Jul;29(4):455-63

32. Munakata R, Yamamoto T, Hosokawa Y, et al. Massive pulmonary embolism requiring extracorporeal life support treated with catheter-based interventions. Int Heart J 2012; 53: 370-374.

33. Foley PJ, Morris RJ, Woo EY, et al. 2010. Limb ischemia during femoral cannulation for cardiopulmonary support. J Vasc Surg 2010 52:850-3.

\section{Legends}

Figure 1. The patient with high-risk PE showing hemodynamic collapse, who were successfully treated with ECMO as an adjunct to EKOS? acoustic pulse thrombolysis. $3.52 \mathrm{~L}$. ECMO flow and $1.72 \mathrm{~L} / \mathrm{min} / \mathrm{m}^{2}$ cardiac index was achieved and the patient stabilized hemodynamically.

Figure 2. ( A) Selective Pulmonary angiography shows a massive pulmonary embolism in the right main pulmonary artery (B) The EKOS? infusion catheter was positioned at the distal edge of the thrombus (C) Post treatment angiography shows complete resolution of clot burden and normal perfusion of the right lung.

Figure 3. (A) Initial computed tomography image shows large saddle thrombus in main pulmonary artery was presented in CT with contrast enhancement. (B) Follow up CT revealed that, pulmonary thrombus in main pulmonary artery was totally disappeared.

\section{Hosted file}

Table 1.docx available at https://authorea.com/users/372324/articles/539735-acousticpulse-thrombolysis-complemented-by-ecmo-improved-survival-in-patients-with-high-riskpulmonary-embolism

\section{Hosted file}

Table 2.docx available at https://authorea.com/users/372324/articles/539735-acousticpulse-thrombolysis-complemented-by-ecmo-improved-survival-in-patients-with-high-riskpulmonary-embolism

\section{Hosted file}

Table 3.doc available at https://authorea.com/users/372324/articles/539735-acousticpulse-thrombolysis-complemented-by-ecmo-improved-survival-in-patients-with-high-riskpulmonary-embolism 

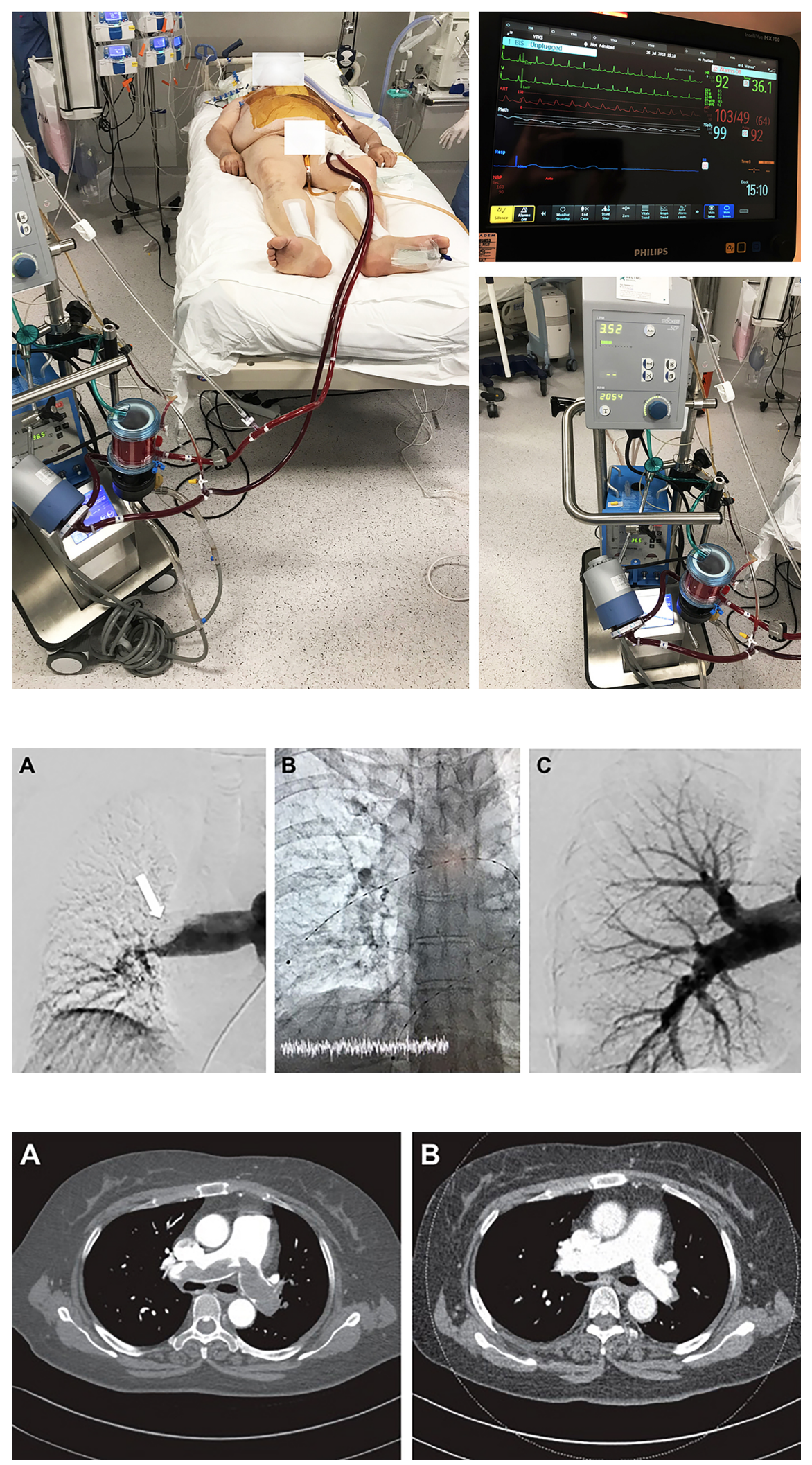